\title{
Hemostatic state of children with type 1 diabetes
}

\author{
Mariano Nicolás Aleman', \\ Elba Irma Díaz', \\ Maria Constanza Luciardi', \\ Ana Carolina Mariani', \\ Maria Cristina Bazán', \\ Adela Victoria Abregu'
}

'Departamento de Bioquímica Aplicada, Facultad de Bioquímica, Universidad Nacional de Tucumán, Tucumán, Argentina

${ }^{2}$ Departamento de Endocrinología, Hospital del Niño Jesús de Tucumán, Tucumán, Argentina
See the commentary "Haemostatic state in children with type 1 diabetes" via https://doi.org/10.6065/apem. 2120071edi01.

Received: 1 July, 2020

Revised: 20 August, 2020

Accepted: 28 September, 2020

\section{Address for correspondence:}

Mariano Nicolás Áleman

Departamento de Bioquímica Aplicada, Facultad de Bioquímica, Universidad Nacional de Tucumán, Balcarce 747, San Miguel de Tucumán, Tucumán 4000, Argentina

Email: mariano_edu@hotmail.com https://orcid.org/0000-0003-30737540
Purpose: Hyperglycemia is one of the factors responsible for the molecular alterations that modify hemostasis. The aim of this study was to determine the levels of circulating molecules that have a prothrombotic impact on the child and adolescent population with type 1 diabetes mellitus.

Methods: There were 35 patients with type 1 diabetes mellitus (11.0 \pm 2.5 years of age and a median $3.7 \pm 2.0$ years of the disease) with no vascular complications and 20 healthy controls with similar age, sex, and body mass index included in the study. The evaluated parameters were fibrinogen, plasminogen activator inhibitor-1 (PAI1), von Willebrand factor antigen, and standard coagulation tests (platelet count, prothrombin time, and activated partial thromboplastin time). Glycemic control was evaluated by hemoglobin A1c and fasting blood glucose tests, and the presence of retinopathy and nephropathy was ruled out. The data obtained were analyzed by IBM SPSS Statistics ver. 20.0 and expressed as mean \pm standard deviation. The Pearson correlation coefficient was applied to investigate correlations between variables.

Results: Diabetic patients showed significantly higher levels of fibrinogen (308 \pm 66 $\mathrm{mg} / \mathrm{dL}$ vs. $246 \pm 18 \mathrm{mg} / \mathrm{dL}, P=0.0001), P A I-1(41.6 \pm 12 \mathrm{ng} / \mathrm{mL}$ vs. $11.7 \pm 1.0 \mathrm{ng} /$ $\mathrm{mL}, P=0.0001)$, and von Willebrand factor antigen $(284 \% \pm 55 \%$ vs. $121 \% \pm 19 \%$, $P=0.0001$ ). However, standard coagulation tests did not show differences between the 2 groups. PAI-1 was correlated with glycemia, hemoglobin A1c, fibrinogen, and von Willebrand factor antigen.

Conclusion: Elevated levels of fibrinogen, PAI-1, and von Willebrand factor antigen were found in the pediatric and adolescent population with type 1 diabetes mellitus, which suggests a prothrombotic state.

Keywords: Type 1 diabetes, Prothrombotic state, Plasminogen activator inhibitor-1, Von Willebrand factor

\section{Highlights}

We conducted a study of haemostatic status of children with T1DM, its change over time and its relationship with the degree of glycaemic control. We found high levels of fibrinogen, plasminogen activator inhibitor-1, von Willebrand factor antigen, a prothrombotic state and micro- and macrovascular complications risk.

\section{Introduction}

Type 1 diabetes mellitus (T1DM) is one of the most common endocrine and metabolic diseases in childhood, and its incidence is increasing rapidly. In 2020, the diabetes burden is expected to double, mainly in young people and in adults with a moderate genetic susceptibility. ${ }^{1)}$

Hyperglycemia can produce molecular changes that alter hemostasis and induce a proinflammatory, prothrombotic, and antifibrinolytic microenvironment. ${ }^{2}$ The main 
components of the hemostatic system affected by hyperglycemia are fibrinogen (Fg), plasminogen activator inhibitor-1 (PAI1), von Willebrand factor antigen (vWF:Ag), and antithrombin. The increase in Fg level has been associated with coronary artery disease in patients with T1DM. Hyperfibrinogenemia causes increase in coagulation activity, clot strength, and blood viscosity and is a marker of inflammatory vascular modifications and endothelial dysfunction. Moreover, Fg contributes to development and activation of atheromatous plaque."

PAI- 1 is present in platelets and is produced by endothelial tissue and adipocytes. It is the main endogenous inhibitor of the fibrinolytic system. A clear association has been observed between te increase in PAI-1 plasma level and prothrombotic diseases such as hypertension, obesity, insulin resistance, and diabetes. $^{4,5)}$

vWF:Ag is a glycoprotein selectively produced by endothelial cells and megakaryocytes. It plays an essential role in hemostasis because it prevents proteolytic degradation of Factor VIII and promotes platelet adhesion and aggregation to damaged endothelium. In addition, Factor VIII mediates vascular inflammation by causing leukocyte recruitment and extravasation and activating the complement cascade. Recently, it has been proposed as a prognostic marker of metabolic and cardiovascular diseases, such as diabetes, myocardial infarction, or stroke. ${ }^{6,7)}$

In diabetic patients, thrombus is more resistant to degradation in the presence of an increase in PAI-1 level. ${ }^{8,9)}$ Although macrovascular complications of T1DM in childhood are rare, endothelial dysfunction and structural anomalies (such as high levels of cell adhesion molecules or increased intima-media thickness in the carotid artery) are present in these patients before the clinical manifestations of vascular disease appear. ${ }^{10-12)}$

The aim of this study was to investigate whether a prothrombotic state exists in children with T1DM by assessing their Fg, PAI-1, and vWF:Ag levels and standard coagulation tests, such as platelet count, prothrombin time (PT), and activated partial thromboplastin time (aPTT). This investigation also attempted to determine if the studied molecules vary with time since T1DM was diagnosed and degree of glycemic control and to analyze the relationships between PAI-1 and the tested parameters.

\section{Materials and methods}

\section{Study design/patient selection}

This observational, analytic, and cross-sectional design included 35 patients with T1DM (20 males and 15 females) who visited the Department of Endocrinology of a pediatric hospital in Tucumán (Argentina) from March 2015 to December 2017. The mean age of diabetic patients was $11.0 \pm 2.5$ years, and the time since diagnosis was $3.7 \pm 2.0$ years, with no clinically demonstrable vascular complications; these patients were compared with 20 controls with similar age, sex, and body mass index (BMI). None of the T1DM patients or the controls had personal or first-degree family history of bleeding, thrombophilia, or thrombosis. All patients underwent a full clinical evaluation, including weight, height, BMI, duration of the disease, and any family history of diabetes and/or cardiovascular disease. Weight was measured using a mechanical platform scale (p-1003-k, Cam, Buenos Aires, Argentina) while the participants wore light clothing and no shoes. Height was determined by stadiometer (Seca 213, Seca, Hamburgo, Germany) while the participants stood with their ankles together, relaxed shoulders, and both arms at their sides. BMI was estimated using Quetelet's Index (weight/size ${ }^{2}$ ). Children with T1DM were being treated with long-acting insulin glargine (Lantus, Sanofi, Bs As, Argentina) at a dosage of $0.7 \pm 0.3 \mathrm{units} / \mathrm{kg} / \mathrm{day}$, and hyperglycemia was corrected with short-acting insulin (Apidra, Sanofi).

Absence of retinopathy was confirmed by ophthalmological evaluation using fundus examination. Nephropathy was ruled out by measuring urine albumin via immunoturbidimetric assay (DCA 2000, Siemens, US).

All children included in this protocol had normal lipid profile and blood pressure levels for their age and sex, according to the American Diabetes Association (ADA) criteria. Patients with inflammatory or infectious diseases, renal and hepatic diseases, acute or chronic diseases, malabsorption disorder, hypothyroidism, or other endocrine diseases were excluded from this protocol.

\section{Biochemical parameters}

Blood samples were obtained between 8 and 10 AM by venipuncture without a tourniquet after overnight fasting. Platelet count was assessed using anticoagulated blood collected with a $10 \%$ ethylenediamine tetraacetic acid additive using a blood cell counter (Sysmex KX 21, Kobe, Japan). Platelet-poor plasma was collected with $3.2 \%(0.109 \mathrm{M})$ sodium citrate at a 1:9 ratio and centrifuged at 3,000 rpm for 15 minutes. PT, aPTT, and Fg tests were immediately carried out using an ACL 300 coagulation analyzer (Diamond Diagnostics, Holliston, MA, USA). The PAI-1 and vWF:Ag plasma levels were measured by enzyme-linked immunosorbent assay (Asserachrom, Diagnostica Stago, Asnières-sur-Seine, France). Glycemic control was evaluated using hemoglobin Alc (HbAlc; DCA 2000, Siemens, USA), fasting blood glucose (enzymatic method, Wiener Lab, Rosario, Argentina), and capillary blood glucose levels. According to the recommendation by the ADA, HbAlc $<8 \%$ value was considered to represent good glycemic control (GGC), while $\mathrm{HbAlc} \geq 8 \%$ indicated poor glycemic control (PGC). ${ }^{13)}$

\section{Statistical analysis}

Statistical analysis was performed using the IBM SPSS Statistics ver. 20.0 (IBM Co., Armonk, NY, USA). A Kolmogorov-Smirnov 
test was used to determine quantitative variable distribution. All data were expressed as frequency and percentage for categorical data and as mean \pm standard deviation or median with Q1 and Q3 for numeric data. Differences in participant characteristics were compared across subgroups using a chi-square test for categorical variables and a $T$-test or Mann-Whitney $U$-test for continuous variables as appropriate. To compare the 2 groups, statistical hypothesis testing was applied for independent samples. The Pearson correlation coefficient was used to investigate correlations between parameters. A $P$-value $<0.05$ was considered statistically significant.

\section{Results}

The clinical and biochemical characteristics of the studied groups are shown in Table 1. Diabetic patients presented with significantly higher values of HbA1c, Fg, PAI-1, and vWF:Ag; however, standard coagulation tests did not show significant differences between the groups.

According to HbAlc value, $73 \%(n=26)$ of children with T1DM had PGC, and 27\% ( $n=9)$ demonstrated GGC. Capillary blood glucose values were used to monitor the patients, who had average readings of $174 \mathrm{mg} / \mathrm{dL}$ before breakfast, $190 \mathrm{mg} /$ $\mathrm{dL}$ before lunch, and $210 \mathrm{mg} / \mathrm{dL}$ before dinner. No significant differences were found in any hemostatic parameters studied between diabetic patients with GGC or PGC (Table 2).

Table 1. Clinical and biochemical characteristics of the groups studied

\begin{tabular}{|c|c|c|c|}
\hline Characteristic & Diabetic $(n=35)$ & Control $(n=20)$ & $P$-value \\
\hline Sex, male:female $(n)$ & $20: 15$ & $13: 7$ & 0.567 \\
\hline Age (yr), median (IQR) & $11(10-13)$ & $10(9-12)$ & 0.137 \\
\hline BMI $\left(\mathrm{kg} / \mathrm{m}^{2}\right)$ & $19.4 \pm 4.0$ & $18.7 \pm 1.5$ & 0.630 \\
\hline Diabetes evolution (yr) & $3.7 \pm 2.0$ & - & - \\
\hline $\mathrm{FBG}(\mathrm{mg} / \mathrm{dL})$ & $210 \pm 109$ & $77 \pm 8$ & $0.0001^{*}$ \\
\hline $\mathrm{HbA1c}(\%)$ & $10.4 \pm 3.0$ & $5.8 \pm 0.4$ & $0.0001^{*}$ \\
\hline $\mathrm{TC}(\mathrm{mg} / \mathrm{dL})$ & $167 \pm 39$ & $156 \pm 28$ & 0.414 \\
\hline HDL-C (mg/dL) & $45 \pm 12$ & $42 \pm 8$ & 0.471 \\
\hline LDL-C (mg/dL) & $107 \pm 39$ & $99 \pm 30$ & 0.569 \\
\hline Triglyceride (mg/dL) & $90 \pm 48$ & $87 \pm 16$ & 0.788 \\
\hline PT $(\%)$ & $86 \pm 15$ & $90 \pm 9$ & 0.468 \\
\hline aPTT (seg) & $45 \pm 6$ & $42 \pm 5$ & 0.163 \\
\hline Platelet count $\left(\times 10^{3} / \mu \mathrm{L}\right)$ & $286 \pm 37$ & $209 \pm 50$ & 0.539 \\
\hline Fibrinogen (mg/dL) & $308 \pm 66$ & $246 \pm 18$ & $0.0001^{*}$ \\
\hline PAl-1 (ng/mL) & $41.6 \pm 12.0$ & $11.7 \pm 1.0$ & $0.0001^{*}$ \\
\hline vWF:Ag (\%) & $284 \pm 55$ & $121 \pm 19$ & $0.0001^{*}$ \\
\hline
\end{tabular}

Values are presented as mean \pm standard deviation unless otherwise indicated.

$I Q R$, interquartile range; $B M I$, body mass index; $F B G$, fasting blood glucose; HbA1c, glycosylated hemoglobin A1c; TC, total cholesterol; HDL-C, high-density lipoprotein cholesterol; LDL-C, low-density lipoprotein cholesterol; PT, prothrombin time; aPTT, activated partial thromboplastin time; PAI-1, plasminogen activator inhibitor-1; vWF:Ag, Von Willebrand Factor:Antigen.

${ }^{*} P<0.05$, statistically significant difference.
Analysis of variables according to duration of disease $\leq 3$ years $(2 \pm 0.7)$ or $>3$ years $(5.4 \pm 1.3)$ did not show differences in any of the studied molecules (Table 3). Our analysis of parameter correlations showed a positive correlation of PAI-1 level with glycemia, HbA1c, Fg, and vWF:Ag (Fig. 1).

\section{Discussion}

Vascular complications are the main causes of morbidity and mortality in diabetes. In patients with T1DM, different alterations of the coagulation system could be of interest, such as alteration in fibrin clot structure, changes in procoagulant molecules concentration, and reduction in fibrinolytic system activity. However, information on coagulation disorders and altered fibrinolysis in children with T1DM is limited. ${ }^{14,15)}$

The normal values for the global tests of PT and aPTT in our patients could be due to the sensitivity of the reagents and the coagulometer used. In children with T1DM, there were no differences in platelet count, PT, and aPTT in comparison with healthy controls; other authors have obtained similar results. ${ }^{16,17)}$

Table 2. Prothrombotic molecules in diabetics according to the degree of glycemic control

\begin{tabular}{lccc}
\hline Variable & GGC $(n=9)$ & PGC $(n=26)$ & $P$-value \\
\hline FBG $(\mathrm{mg} / \mathrm{dL})$ & $123.13 \pm 62.51$ & $253.44 \pm 102.96$ & $0.004^{*}$ \\
HbA1c $(\%)$ & $7.2 \pm 0.3$ & $11.5 \pm 2.7$ & $0.0001^{*}$ \\
PT $(\%)$ & $88 \pm 8$ & $84 \pm 18$ & 0.636 \\
aPTT $(\mathrm{sec})$ & $45 \pm 3$ & $46 \pm 8$ & 0.619 \\
Platelet count $\left(\times 10^{3} / \mu \mathrm{L}\right)$ & $274 \pm 47$ & $290 \pm 36$ & 0.492 \\
Fibrinogen $(\mathrm{mg} / \mathrm{dL})$ & $341 \pm 64$ & $290 \pm 63$ & 0.056 \\
PAl-1 $(\mathrm{ng} / \mathrm{mL})$ & $43.8 \pm 12.1$ & $40.4 \pm 13.3$ & 0.545 \\
vWF:Ag $(\%)$ & $272 \pm 39$ & $288 \pm 58$ & 0.630 \\
\hline Values
\end{tabular}

Values are presented as mean \pm standard deviation.

GGC, good glucemic control ( $\mathrm{HbA} 1 \mathrm{c} \leq 8 \%)$; PGC, poor glucemic control (HbA1c>8\%). FBG, fasting blood glucose; HbA1c, glycosylated hemoglobin A1c; PT, prothrombin time; aPTT, activated partial thromboplastin time; PAI-1, plasminogen activator inhibitor-1; vWF:Ag, Von Willebrand Factor:Antigen.

${ }^{*} P<0.05$, statistically significant difference.

Table 3. Prothrombotic molecules in diabetics according to the time of evolution of the disease

\begin{tabular}{lccc}
\hline Variable & $\leq 3$ years $(n=16)$ & $>3$ years $(n=19)$ & $P$-value \\
\hline FBG $(\mathrm{mg} / \mathrm{dL})$ & $208.9 \pm 123.7$ & $210.79 \pm 103.5$ & 0.968 \\
HbA1c $(\%)$ & $9.94 \pm 3.08$ & $10.72 \pm 3.05$ & 0.566 \\
PT $(\%)$ & $83 \pm 12$ & $88 \pm 18$ & 0.236 \\
aPTT $(\mathrm{sec})$ & $43 \pm 4$ & $47 \pm 7$ & 0.130 \\
Platelet count $\left(\times 10^{3} / \mu \mathrm{L}\right)$ & $288 \pm 40$ & $283 \pm 35$ & 0.937 \\
Fibrinogen $(\mathrm{mg} / \mathrm{dL})$ & $320 \pm 73$ & $297 \pm 60$ & 0.253 \\
PAl-1 $(\mathrm{UA} / \mathrm{mL})$ & $46.1 \pm 11.4$ & $38.6 \pm 11.9$ & 0.168 \\
vWF:Ag $(\%)$ & $264 \pm 50$ & $293 \pm 60$ & 0.377 \\
\hline Values
\end{tabular}

Values are presented as mean \pm standard deviation.

FBG, fasting blood glucose; HbA1c, glycosylated hemoglobin A1c; PT, prothrombin time; aPTT, activated partial thromboplastin time; PAI-1, plasminogen activator inhibitor-1; vWF:Ag, Von Willebrand Factor:Antigen. 

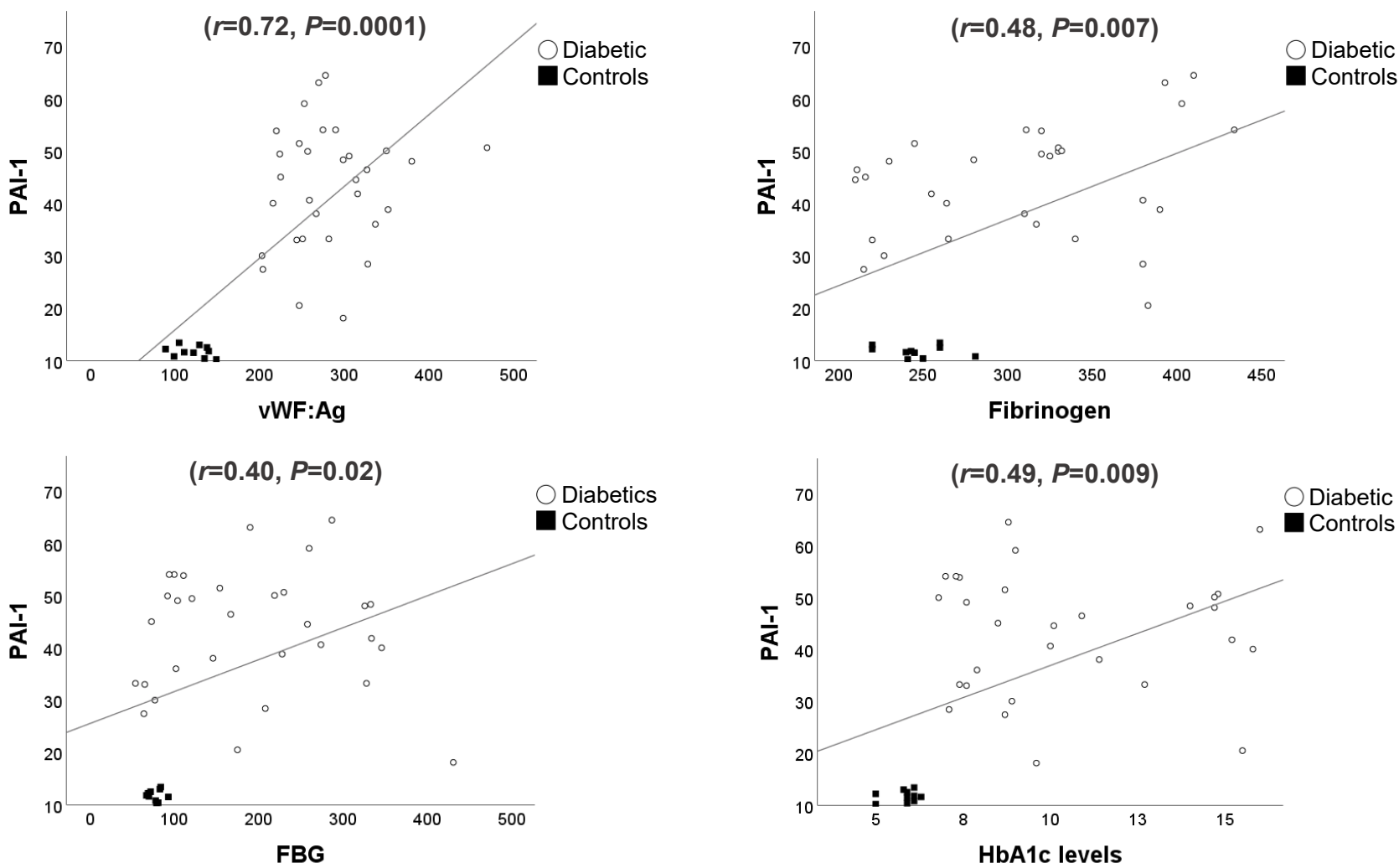

Fig. 1. Scatter plots showing the relationship between the PAl-1 and other variables. PAl-1, plasminogen activator inhibitor-1; vWF:Ag, Von Willebrand Factor:Antigen; FBG, fasting blood glucose; $\mathrm{HbA1c}$, glycosylated hemoglobin A1c. The Pearson correlation coefficient $(r)$ and the $P$-value are shown at the top of each graph. A $P<0.05$ was considered significant.

However, in diabetic adults, some authors have reported only shortening of aPTT, while others found shortening of PT; all agree that there is platelet dysfunction in diabetics in comparison to nondiabetic individuals. ${ }^{18-20)}$

The most interesting results of this study were the elevated levels of Fg, PAI-1, and vWF:Ag in diabetic children. Several investigations in adults and children with T1DM have suggested abnormalities in hemostasis. Carmassi et al. ${ }^{16)}$ found that patients with T1DM had elevated levels of Fg, Factor VII, thrombin and antithrombin complexes, and PAI-1, with a reduction in tissue plasminogen activator (tPA) activity in comparison to controls. Targher et al. ${ }^{21)}$ reported that, in comparison to healthy controls, the plasma levels of Fg, vWF:Ag, soluble intercellular adhesion molecule-1, and PAI-1 were remarkably higher in young people with T1DM, with no clinical evidence of macrovascular disease.

All these studies indicate hemostatic disorders in patients with T1DM. Several mechanisms contribute to the prothrombotic state in these patients, including endothelial dysfunction, hypercoagulability, and hypofibrinolysis. Further, chronic hyperglycemia promotes glycation of the proteins involved in fibrinolysis and affects clot structure, increasing its resistance to degradation by plasmin. ${ }^{22,23)}$ Moreover, hyperglycemia activates the metabolic pathways of polyol and protein kinase C, which increases oxidative stress. All these processes cause vascular damage that affects the hemostatic system. ${ }^{24,25)}$

Many researchers have emphasized the importance of GGC, particularly as diabetes duration increases, to reduce the risk of vascular complications. ${ }^{26)}$ Over time, the presence of PGC alters the levels of some metabolites, such as lipid profile (total cholesterol, triglycerides, high-density lipoprotein cholesterol, low-density lipoprotein cholesterol), creatinine, uric acid, and some cytokines (interleukin [IL]-6, IL-17, interferon- $\gamma$ ). ${ }^{27-29)}$ However, changes in the hemostatic system have not been well studied in children. In this work, degree of glycemic control and duration of T1DM diagnosis did not significantly affect the hemostatic parameters evaluated. Similar findings were reported in a study of 84 Turkish T1DM children with a disease duration longer than 5 years, which showed that there was no correlation between serum PAI-1 level and disease duration or presence of microvascular complications. ${ }^{30)}$ In contrast, Zhao et al. ${ }^{31)}$ reported that PGC in adults with T1DM was correlated with increased Fg and shortening of PT and aPTT. Moreover, recent studies in young adults with T1DM found that chronic hyperglycemia and elevated $\mathrm{HbAlc}$ level were correlated with increased PAI-1, platelet aggregation, and protein C levels. ${ }^{32)}$ This divergence between glycemic control and hemostatic molecule levels suggests that more time is necessary to observe a change, or that there are other factors involved in adults (obesity, 
smoking, hypertension), which highlights the importance of GGC through the disease.

Fibrinolysis is a normal body process that remodels or degrades a blood clot. Fibrinolysis is activated by conversion of inactive plasminogen to plasmin (the enzyme responsible for cleaving fibrin) on the thrombus or cell surfaces by serine proteases such as tPA or urokinase plasminogen activator. Therefore, fibrinolysis is regulated highly. ${ }^{33)}$ This regulation is carried out primarily by PAI-1, which inhibits tPA. A systematic review of 52 epidemiological studies supported a relationship between PAI- 1 and diabetes, which shows its potential as a biomarker of prothrombotic risk in this disease. ${ }^{34,35)}$ Our findings of elevated PAI-1 in children with T1DM and its positive correlation with levels of fasting blood glucose, HbAlc, Fg, and vWF:Ag suggest that elevated PAI-1 contributes to the hypercoagulability state seen in T1DM.

This work had some limitations. It was a cross-sectional design, which only allows for associations but no causality. The sample size was relatively small, and some results cannot be applied indiscriminately. Therefore, further studies are necessary to confirm our results and evaluate the clinical usefulness of these biomarkers to identify prothrombotic risk in diabetic children.

In brief, elevated levels of Fg, PAI-1, and vWF:Ag in pediatric patients with T1DM suggest a prothrombotic state. Children with T1DM face many years of hyperglycemia and the risk of early micro- and macrovascular complications. Therefore, an early detection of higher levels of these molecules could prevent or delay alterations beginning in childhood.

\section{Ethical statement}

This investigation protocol was approved by the Education and Investigation Committee of Tucumán (CEI ethics committee, Tucumán, Argentina. Exp No.1699-410), and written informed consent was obtained from the parents and/or tutors of the patients.

\section{Conflict of interest}

No potential conflict of interest relevant to this article was reported.

\section{Acknowledgments}

This study was funded by grants of the Science and Art Innovation Technology Secretary of the Universidad Nacional de Tucumán and the Argentine Diabetes Society.

\section{References}

1. Vehik K, Dabelea D. The changing epidemiology of type 1 diabetes: why is it going through the roof? Diabetes Metab
Res Rev 2011;27:3-13.

2. Targher G, Chonchol M, Zoppini G, Franchini M. Hemostatic disorders in type 1 diabetes mellitus. Semin Thromb Hemost 2011;37:58-65.

3. Rodrigues TC, Snell-Bergeon JK, Maahs DM, Kinney GL, Rewers M. Higher fibrinogen levels predict progression of coronary artery calcification in adults with type 1diabetes. Atherosclerosis 2010;210:671-3.

4. Alessi MC, Poggi M, Juhan-Vague I. Plasminogen activator inhibitor-1, adipose tissue and insulin resistance. Curr Opin Lipidol 2007;18:240-5.

5. Cesari M, Pahor M, Incalzi RA. Plasminogen activator inhibitor-1 (PAI-1): a key factor linking fibrinolysis and age-related subclinical and clinical conditions. Cardiovasc Ther 2010;28:72-91.

6. Gragnano F, Sperlongano S, Golia E, Natale F, Bianchi $\mathrm{R}$, Crisci M, et al. The role of von Willebrand Factor in vascular inflammation: from pathogenesis to targeted therapy. Mediators Inflamm 2017;2017:5620314.

7. Frankel DS, Meigs JB, Massaro JM, Wilson PW, O'Donnell CJ, D'Agostino RB, et al. Von Willebrand Factor, type 2 diabetes mellitus, and risk of cardiovascular disease: The Framingham Offspring Study. Circulation 2008;118:25339.

8. Madan R, Gupt B, Saluja S, Kansra UC, Tripathi BK, Guliani BP. Coagulation profile in diabetes and its association with diabetic microvascular complications. J Assoc Physicians India 2010;58:481-4.

9. Adly AA, Elbarbary NS, Ismail EA, Hassan SR. Plasminogen activator inhibitor-1 (PAI-1) in children and adolescents with type 1 diabetes mellitus: relation to diabetic microvascular complications and carotid intima media thickness. J Diabetes Complications 2014;28:340-7.

10. Carrizo T del R, Prado MM, Velarde MS, Díaz EI, Bazán MC, Abregú AV. Soluble E-Selectin in children and adolescents with type 1 diabetes. Medicina (B Aires) 2008;68:193-7.

11. Rabago Rodríguez R, Gómez-Díaz RA, Tanus Haj J, Avelar Garnica FJ, Ramirez Soriano E, Nishimura Meguro E, et al. Carotid intima-media thickness in pediatric type 1 diabetic patients. Diabetes Care 2007;30:2599-602.

12. Babar GS, Zidan H, Widlansky ME, Das E, Hoffmann RG, Daoud M, et al. Impaired endothelial function in preadolescent children with type 1 diabetes. Diabetes Care 2011;34:681-5.

13. American Diabetes Association. 13. Children and adolescents: standards of medical care in diabetes-2020. Diabetes Care 2020;43(Suppl 1):S163-82.

14. Romano M, Pomilio M, Vigneri S, Falco A, Chiesa PL, Chiarelli F, et al. Endothelial perturbation in children and adolescents with type 1 diabetes: association with markers of the inflammatory reaction. Diabetes Care 2001;24:16748.

15. Sobczak A, Stewart AJ. Coagulatory defects in type-1 and type-2 diabetes. Int J Mol Sci 2019;20:6345. 
16. Carmassi F, Morale M, Puccetti R, De Negri F, Monzani F, Navalesi R, et al. Coagulation and fibrinolytic system impairment in insulin dependent diabetes mellitus. Thromb Res 1992;67:643-54.

17. Binay C, Bozkurt Turhan A, Simsek E, Bor O, Akay OM. Evaluation of coagulation profile in children with type 1 diabetes mellitus using rotational thromboelastometry. Indian J Hematol Blood Transfus 2017;33:574-80.

18. Blann AD, Lip GY. Endothelial integrity, soluble adhesion molecules and platelet markers in type 1 diabetes mellitus. Diabet Med 1998;15:634-42.

19. Sapkota B, Shrestha SK, Poudel S. Association of activated partial thromboplastin time and fibrinogen level in patients with type II diabetes mellitus. BMC Res Notes 2013;6:485.

20. Kim HK, Kim JE, Park SH, Kim YI, Nam-Goong IS, Kim ES. High coagulation factor levels and low protein C levels contribute to enhanced thrombin generation in patients with diabetes who do not have macrovascular complications. J Diabetes Complications 2014;28:365-9.

21. Targher G, Bertolini L, Zoppini G, Zenari L, Falezza G. Increased plasma markers of inflammation and endothelial dysfunction and their association with microvascular complications in type 1 diabetic patients without clinically manifest macroangiopathy. Diabet Med 2005;22:999-1004.

22. Behl T, Velpandian T, Kotwani A. Role of altered coagulation-fibrinolytic system in the pathophysiology of diabetic retinopathy. Vascul Pharmacol 2017;92:1-5.

23. Lemkes BA, Hermanides J, Devries JH, Holleman F, Meijers JC, Hoekstra JB. Hyperglycemia: a prothrombotic factor? J Thromb Haemost 2010;8:1663-9.

24. Milne R, Brownstein S. Advanced glycation end products and diabetic retinopathy. Amino Acids 2013;44:1397-407.

25. Geraldes P, King GL. Activation of protein kinase C isoforms and its impact on diabetic complications. Circ Res 2010;106:1319-31.

26. Daniels M, DuBose SN, Maahs DM, Beck RW, Fox LA, Gubitosi-Klug R, et al. Factors associated with microalbuminuria in 7,549 children and adolescents with type 1 diabetes in the T1D Exchange clinic registry.
Diabetes Care 2013;36:2639-45.

27. Mohammad HA, Farghaly HS, Metwalley KA, Monazea EM, Abd El-Hafeez HA. Predictors of glycemic control in children with Type 1 diabetes mellitus in Assiut-Egypt. Indian J Endocrinol Metab 2012;16:796-802.

28. Dutta T, Kudva YC, Persson XM, Schenck LA, Ford GC, Singh RJ, et al. Impact of long-term poor and good glycemic control on metabolomics alterations in type 1 diabetic people. J Clin Endocrinol Metab 2016;101:1023-33.

29. Franco MM, Morales MMM, Duarte PM, Napimoga $\mathrm{MH}$, Benatti BB. Glycemic control and the production of cytokines in diabetic patients with chronic periodontal disease. RGO - Revista Gaúcha de Odontologia 2015;63: 432-8.

30. Kırmızıbekmez H, Güven A, Yıldız M, Dursun F, Cebeci N, Hancili S. Detection of vascular risk markers in children and adolescents with type 1 diabetes. J Pediatr Endocrinol Metab 2014;27:87-92.

31. Zhao Y, Zhang J, Zhang J, Wu J. Diabetes mellitus is associated with shortened activated partial thromboplastin time and increased fibrinogen values. PLoS One 2011;6: e16470.

32. Sarkisova K, Jarek-Martynowa IR, Shestakova M, Chirkova L, Nikankina L, Shamkhalova M. Effect of HbA1c and hyperglycemia on hemostasis in patients with type 1 diabetes. Endocrine Abstracts 2019;63:GP218.

33. Zorio E, Gilabert-Estelles J, Espana F, Ramón LA, Cosín R, Estellés A. Fibrinolysis: the key to new pathogenetic mechanisms. Curr Med Chem 2008;15:923-9.

34. Singh NK, Gupta A, Behera DR, Dash D. Elevated plasminogen activator inhibitor type-1 (PAI-1) as contributing factor in pathogenesis of hypercoagulable state in antiphospholipid syndrome. Rheumatol Int 2013;33:23316.

35. Yarmolinsky J, Bordin Barbieri N, Weinmann T, Ziegelmann PK, Duncan BB, Inês Schmidt M. Plasminogen activator inhibitor- 1 and type 2 diabetes: a systematic review and meta-analysis of observational studies. Sci Rep 2016;6:17714. 